\title{
A Prospective Study of Conventional Transbronchial Needle Aspiration: Performance and Cost Utility
}

\author{
A.R.L. Medford S. Agrawal C.M. Free J.A. Bennett \\ Department of Respiratory Medicine, Glenfield Hospital, University Hospitals of Leicester NHS Trust, Leicester, UK
}

\section{Key Words}

Cost analysis - Diagnosis - Lung cancer - Mediastinoscopy •

Staging $\cdot$ Transbronchial needle aspiration

\begin{abstract}
Background: Conventional transbronchial needle aspiration (TBNA) is a cheap, minimally invasive tool for lung cancer staging and diagnosis. Endobronchial ultrasound-guided TBNA (EBUS-TBNA) is more sensitive but is more expensive and less widely available. We describe a prospective analysis of TBNA diagnostic, staging and cost utility in a centre in the UK. Objectives: To illustrate the potential diagnostic, staging and cost utility of a low cost conventional TBNA service. Methods: A prospective analysis of 79 TBNA procedures over a 2-year period was performed looking at performance and cost utility in a 'mixed' cohort with variable pretest probability of malignancy (year 1 ) followed by a high probability cohort (year 2). Results: TBNA avoided mediastinoscopy in $25 \%$ of the cases overall (37\% in high probability vs. $13 \%$ in the 'mixed' cohort, $p=0.03$ ). The overall prevalence of malignancy was $84 \%$, sensitivity $79 \%$, negative predictive value $58 \%$ and accuracy $85 \%$. Diagnostic utility varied with pre-test probability and nodal station. TBNA downstaged $8 \%$ of lung cancer patients to receive surgery and confirmed the pre-treatment stage (inoperability) in $74 \%$.
\end{abstract}

TBNA led to theoretical cost savings of GBP 560 per patient. Conclusions: TBNA can achieve a high diagnostic sensitivity for cancer in high probability patients and stage the majority appropriately, thereby avoiding unnecessary mediastinoscopies and reducing costs. It may also down-stage a minority to have surgery. TBNA is cheap, routinely available and learnable. As EBUS-TBNA will take time to develop due to its costs, all respiratory centres should perform TBNA at flexible bronchoscopy in suspected lung cancer with accessible mediastinal adenopathy. Copyright $\odot 2010$ S. Karger AG, Basel

\section{Introduction}

Conventional transbronchial needle aspiration (TBNA) is an established procedure for the diagnosis and staging of lung cancer. A recent systematic review indicated it had a pooled sensitivity of $76 \%$, and it has been used to diagnose benign conditions in some case series [1, 2]. However, this systematic review was not restricted to non-small cell lung cancer (NSCLC), and did not control for study quality. A previous meta-analysis of conventional TBNA which controlled for these factors demonstrated the performance of TBNA is highly variable, with a pooled sensitivity varying from 39 to $78 \%$ depending on

\section{KARGER}

Fax +41613061234

E-Mail karger@karger.ch

www.karger.com (c) 2010 S. Karger AG, Basel

0025-7931/10/0796-0482\$26.00/0

Accessible online at:

www.karger.com/res
Dr. Andrew R.L. Medford

North Bristol Lung Centre

Southmead Hospital, Westbury-on-Trym

Bristol BS10 5NB (UK)

Tel. +44 117959 5284, Fax +44 117959 5863, E-Mail andrewmedford@ hotmail.com 
whether the disease prevalence of mediastinal metastasis is low (34\%) or high (81\%), respectively [3]. In addition, if a more stringent definition of exact histological diagnosis (rather than malignancy per se) is used, the performance of TBNA inevitably drops but is still $50 \%$ and therefore worthwhile [4].

The advantage of conventional TBNA is that it is a rapid, learnable technique with a short learning curve [5] and can yield a pathological diagnosis immediately if onsite cytopathology is used. In addition, it is well tolerated by patients and is as acceptable as flexible bronchoscopy alone [6]. TBNA can also reduce the requirement for more expensive and invasive staging procedures such as mediastinoscopy or re-staging mediastinoscopy in 35\% of the patients in 2 studies [7, 8].

CT-guided transthoracic needle aspiration is a less invasive alternative to mediastinoscopy with $84 \%$ overall sensitivity in staging hilar and mediastinal nodes in those with lung cancer following a negative flexible bronchoscopy [9]. This, however, has the potential risks of pneumothorax and bleeding as well as requiring specialist expertise and equipment. As it is more invasive than conventional TBNA, its role may be complementary to TBNA.

Endobronchial ultrasound-guided TBNA (EBUSTBNA) offers a superior yield to conventional TBNA [10], which may relate to both nodal motion and varying distance between carina and nodal position only evident in real-time imaging [11]. Breathing-induced nodal motion in the mediastinum can be substantial. Using repeat CT or fluoroscopy on calcified nodes, one study has illustrated the need for an extra 5-mm margin for radiotherapy fields [12] with another showing an almost 7-mm variation in craniocaudal nodal position [13]. This has importance in radiotherapy planning for lung cancer and lymphoma [14]. However, EBUS-TBNA is significantly more expensive in terms of capital and running costs and also requires operators to undertake specific training with a different bronchoscope. Therefore, EBUS-TBNA is only likely to be used in selected centres with both sufficient funds and the requisite skills available. However, conventional TBNA utilises the same flexible bronchoscope with low-cost TBNA needles and is available to all centres with respiratory physicians performing flexible bronchoscopy.

It is always preferable to utilise techniques that are minimally invasive and also reduce costs, and this is especially so at this time of economic recession and limitations on funding. In this setting, conventional TBNA has the potential to have a greater impact on reducing the need for surgical staging as it can be performed in a far greater number of centres than EBUS-TBNA. TBNA does, however, require a period of training lasting for approximately 50 procedures - even for bronchoscopists who have performed 300 normal procedures [15] - and results improve with time [16], especially if at least 4 passes are taken per node [17]. At present, in the UK only a limited number of centres are performing TBNA (27\% in a survey in 2002 [18]).

In 2006 the University Hospitals of Leicestershire National Health Service Trust, established conventional TBNA as part of routine practice (if deemed clinically helpful) at bronchoscopy for evaluation of $\mathrm{N} 2$ and N3 disease to assist with staging and diagnosis of lung cancer. The Trust does have a thoracic surgical unit but conventional TBNA was established in part in an attempt to reduce the demand for mediastinal surgical staging in cases where a positive malignant diagnosis was achieved by conventional TBNA and the cancer was non-resectable. Furthermore, no EBUS-TBNA service was available locally.

We examined the diagnostic and staging utility of conventional TBNA for lung cancer in a 2 -year prospective analysis in 2 sequential cohorts of patients with either 'mixed' or high clinical pre-test probability of lung cancer and performed a cost analysis. We assessed the relationship between pre-test clinical probability of malignancy, nodal location and performance of conventional TBNA at the time.

\section{Methods}

A prospective audit of all conventional TBNA procedures $(\mathrm{n}=$ 79) was performed over a 2-year period (July 2006 to July 2008). In the initial period (July 2006 to July 2007), TBNA was being utilised to assist in the diagnosis of unexplained mediastinal adenopathy (including non-malignant disease) in an unselected cohort as well as diagnosis and staging of lung cancer with suspected N2 and N3 disease. Thus, the pre-test clinical probability of malignancy was intermediate and this cohort was defined as having a 'mixed' pre-test probability. In the latter context, a positive TBNA diagnosis of malignancy was accepted as accurate but a negative TBNA was followed by mediastinoscopy to clarify if this was a false negative TBNA (positive at mediastinoscopy for malignancy) or a true negative TBNA for malignancy (negative at mediastinoscopy for malignancy but positive for benign disease). For true negative TBNA results for malignancy, it was also noted whether the TBNA gave a specific benign diagnosis or just benign cytology. From July 2007 to July 2008, only cases with a high pretest clinical probability of malignancy were selected for TBNA. Data were compared between the first year $(n=38)$ of 'mixed' pretest clinical probability of lung cancer and the second year (high pre-test probability of lung cancer, $n=41$ ). 
Conventional TBNA was performed using disposable 21gauge cytology needles (NA-601D-1519; Olympus Keymed, Southend-on-Sea, UK) by the same 3 experienced conventional TBNA bronchoscopists (S.A., J.A.B., C.M.F.) with 4 passes per nodal station via a standard flexible bronchoscope (Olympus Keymed). Only N2 or N3 lymph nodes with a short-axis diameter greater or equal to $1 \mathrm{~cm}$ on CT were sampled by TBNA, and all nodes were between 1 and $2 \mathrm{~cm}$ in size. Paratracheal and subcarinal stations were sampled in this study, although there were no pre-selection criteria in this study (see Discussion). Rapid on-site cytology was not available. Specimens were put into liquid cytology preparation for flush solution, cytocentrifugation, cell pellet resuspension then staining and cytopathological analysis rather than using the recommended smear technique, which is thought to have less negative influence on the cellular architecture and structure of granulomas [19-21]. The liquid cytology method has been the long-standing preference of the local pathologists and was therefore used in this study. Ethical approval was obtained from our local institutional review board, the Leicestershire, Northamptonshire and Rutland Research Ethics Committee.

A cost analysis was performed comparing the conventional TBNA route (HRG D07) and other necessary procedures, for example CT-guided lung biopsy (CTBx) (HRG D34) and MED (HRG D05) with the theoretical diagnostic pathway if TBNA had not been utilised. Diagnostic utility, CT and final staging, final diagnosis, treatment and cost analysis were assessed in both cohorts as well as a separate assessment of diagnostic utility in nodal locations.

Data were analysed using Graph Pad Prism version 4 software. Normality testing was assessed by the Ryan-Joiner test. Means and standard error (SE) are quoted for normal data. Medians and inter-quartile range (IQR) in parentheses are quoted for nonparametric data. Two-column data were analysed with either MannWhitney (nonparametric data) or t test (normal data); multiple comparisons were analysed with either Kruskal-Wallis (nonparametric data) with Dunn's post-test correction or ANOVA (normal data) with Bonferroni post-test correction. Contingency table data were analysed with Fisher's exact test or $\chi^{2}$ test as appropriate and represented by odds ratios (OR) with $95 \%$ confidence intervals $(95 \% \mathrm{CI})$ where possible. In all cases, a p value less than 0.05 was deemed significant.

\section{Results}

\section{Diagnosis}

The average number of patients undergoing TBNA was 38 per year over the whole study period, increasing to 50 per year for the last 7 months. The mean (SE) age was 63.8 years (1.29) with a male preponderance (61\% male, $39 \%$ female), and the nodal locations sampled were subcarinal (81\%) and paratracheal (19\%). In 20 patients (25\%), TBNA was the sole diagnostic modality. Thus, 20 mediastinoscopies were avoided $[15$ patients $(37 \%)$ in the high probability group vs. 5 patients (13\%) in the 'mixed' group, OR $3.81,95 \%$ CI $1.22-11.9, \mathrm{p}=0.03$, Fisher's exact test]. There were no complications or deaths related to TBNA.
Table 1. Diagnostic utility of TBNA and prevalence of malignancy according to pre-test probability of malignancy

\begin{tabular}{llll}
\hline Diagnostic utility & Overall & $\begin{array}{l}\text { 'Mixed' } \\
\text { probability } \\
(\mathrm{n}=38)\end{array}$ & $\begin{array}{l}\text { High } \\
\text { probability } \\
(\mathrm{n}=41)\end{array}$ \\
\hline Sensitivity, \% & 78.7 & 66.7 & 86.4 \\
Negative predictive value, \% & 58.1 & 63.6 & 44.4 \\
Accuracy, \% & 84.6 & 78.9 & 87.8 \\
Prevalence, \% & 83.6 & 71.1 & 95.1 \\
\hline
\end{tabular}

The diagnostic utility of TBNA varied according to the pre-test probability of lung cancer (table 1). In the 'mixed' cohort, both sensitivity and accuracy were lower than in the high pre-test probability cohort, although the negative predictive value was superior for malignancy. Diagnostic utility also varied with nodal sample location. Overall, there were minimal differences in performance for subcarinal and paratracheal TBNA. For patients with 'mixed' pre-test probability of lung cancer, the diagnostic yield of paratracheal TBNA diagnostic utility was far lower than subcarinal TBNA (table 2).

The final diagnosis for the 79 patients is shown in table 3 according to the TBNA result. SCLC was less likely to lead to a false negative TBNA result although this did not reach significance $(15 \%$ false negative vs. $42 \%$ true positive, OR 3.9, 95\% CI 0.99-1.61, $\mathrm{p}=0.11$ ). TBNA gave a false negative result of malignancy in 13 patients. Four patients were diagnosed on endobronchial biopsy of visible tumour and 4 patients on CT-guided lung biopsy each (in both cases the nodes were assumed to be involved), 3 patients were clinically diagnosed with NSCLC (unfit for mediastinoscopy), 1 patient by mediastinoscopy and 1 patient at surgery, indicating a microscopic focus of nodal metastasis which had also not been detected at mediastinoscopy. For patients with benign disease and a true negative TBNA for malignancy, the TBNA yielded benign cytology but did not give a specific diagnosis (of tuberculosis or sarcoidosis for example; table 3).

Of the 62 patients with lung malignancy, treatment details were available for 55 (89\%). Of these 55 patients, $50(91 \%)$ received active anti-cancer treatment: 5 (9\%) surgery, 33 (60\%) chemotherapy, 12 (22\%) radiotherapy. Pre-test clinical probability did not affect the difference in proportion receiving oncological or surgical treatments, of those with proven lung malignancy (data not shown, $\left.\mathrm{p}=0.84, \chi^{2}\right)$. 
Table 2. Diagnostic utility of TBNA

a According to nodal station only

\begin{tabular}{lll}
\hline Diagnostic utility & $\begin{array}{l}\text { Subcarinal } \\
(\mathrm{n}=64)\end{array}$ & $\begin{array}{l}\text { Paratracheal } \\
(\mathrm{n}=15)\end{array}$ \\
\hline Sensitivity, \% & 79.6 & 75.0 \\
Negative predictive value, \% & 60.0 & 50.0 \\
Accuracy, \% & 84.4 & 85.0 \\
\hline
\end{tabular}

b According to nodal station and pre-test probability

\begin{tabular}{|c|c|c|c|c|}
\hline \multirow[t]{2}{*}{ Diagnostic utility } & \multicolumn{2}{|l|}{ Subcarinal } & \multicolumn{2}{|l|}{ Paratracheal } \\
\hline & $\begin{array}{l}\text { 'mixed' probability } \\
(\mathrm{n}=33)\end{array}$ & $\begin{array}{l}\text { high probability } \\
(\mathrm{n}=31)\end{array}$ & $\begin{array}{l}\text { 'mixed' probability } \\
(\mathrm{n}=5)\end{array}$ & $\begin{array}{l}\text { high probability } \\
(\mathrm{n}=10)\end{array}$ \\
\hline Sensitivity, \% & 72.7 & 85.2 & 0.0 & 90.0 \\
\hline Negative predictive value, $\%$ & 64.7 & 50.0 & 60.0 & 0.0 \\
\hline Accuracy, $\%$ & 81.8 & 87.1 & 60.0 & 90.0 \\
\hline
\end{tabular}

\section{Staging}

The impact of TBNA on pre-treatment staging for the 62 patients with lung cancer is shown in table 4. TBNA excluded pN2 disease and down-staged 5 patients (8\%) with suspected $\mathrm{N} 2$ disease (confirmed by pN0 at mediastinoscopy) allowing them to undergo surgical resection. TBNA confirmed $\mathrm{pN} 2$ disease in 46 patients (74\%) and therefore confirmed pre-treatment staging so that these patients did not undergo radical treatment. In the remaining 10 patients (16\%), TBNA gave a false negative result and therefore had no impact on staging. In patients with stage 4 disease, the role of TBNA was for diagnosis (rather than staging) as the metastatic site was not always accessible.

\section{Cost Analysis}

In the context of England's publicly funded National Health Service (NHS) and Payment by Results (the system of internal payments used by the NHS) [22], TBNA is classed as the same procedure as standard fibreoptic bronchoscopy (HRG D07), with CTBx (HRG D34) and mediastinoscopy (HRG D05) classified in other Healthcare Resource Groups (codes used by the NHS to indicate the resources consumed by a particular procedure). The costs to the NHS of each procedure are in table 5 (the cost of the disposable TBNA needles is GBP 38 per patient). Using the 2007/8 National Tariff data [23], a cost analysis of the 79 patients undergoing TBNA (and/or other necessary procedures, e.g. CTBx and mediastinoscopy) is shown in
Table 3. Final diagnosis of patients according to TBNA test performance for mediastinal malignancy

\begin{tabular}{lc}
\hline Final diagnosis & $\mathrm{n}(\%)$ \\
\hline True positive TBNA $(\mathrm{n}=48)$ & $26(54)$ \\
$\quad$ NSCLC & $20(42)$ \\
SCLC & $2(4)$ \\
$\quad$ Metastatic (non-lung) & $9(69)$ \\
False negative TBNA $(\mathrm{n}=13)$ & $2(15)$ \\
$\quad$ NSCLC & $2(15)$ \\
SCLC & $7(39)$ \\
Metastatic (non-lung) & $5(28)$ \\
True negative TBNA ( $=18)^{\mathrm{a}}$ & $3(17)$ \\
$\quad$ Sarcoidosis & $2(11)$ \\
$\quad$ NSCLC (pN0 confirmed at surgery) & $1(6)$ \\
$\quad$ Pneumonia & \\
TB & \\
Schwannoma & \\
\hline & a Benign diagnoses for true negative TBNA confirmed by oth- \\
er techniques as TBNA revealed benign cytology only and not a \\
specific benign diagnosis.
\end{tabular}

table 6 according to pre-test probability. This includes an analysis of the predicted costs had TBNA not been utilised, that is the extra cost of 20 mediastinoscopies (as all would have required mediastinoscopy in the absence of TBNA). The majority of the cost saving is for patients in the high probability cohort undergoing TBNA (table 6). 
Table 4. Impact of TBNA on pre-treatment staging for patients with lung cancer

\begin{tabular}{lll}
\hline False negative TBNA & Effect & Number $(\mathrm{n}=10)$ \\
\hline Stage 3A & No change & 2 \\
Stage 3B & No change & 4 \\
Stage 4 & No change & 3 \\
Not available & No change & 1 \\
\hline True positive TBNA & Effect & Number $(\mathrm{n}=46)$ \\
\hline Stage 3A & Confirmed pN2 & 18 \\
Stage 3B & Confirmed pN2 & 11 \\
Stage 4 & Confirmed pN2 & Number $(\mathrm{n}=5)$ \\
\hline True negative TBNA & Effect & 4 \\
\hline Stage 3A & Excluded pN2 (pN0 at mediastinoscopy and surgery) & 1 \\
Stage 3B & Excluded pN2 (pN0 at mediastinoscopy and surgery) & 1 \\
\hline
\end{tabular}

\section{Discussion}

Our data demonstrate a significant impact of TBNA in diagnosis, staging and cost benefit, by removing the need for 20 mediastinoscopies (25\% of the overall cohort and $37 \%$ of the high probability cohort), confirming pN2 disease unsuitable for radical treatment in $74 \%$ and down staging $8 \%$ who were suitable for surgical resection (confirmed at mediastinoscopy). Ninety-one percent of those with malignancy received anti-cancer treatment implying the indications for TBNA (for suspected malignancy) were appropriate. Our 37\% surgical staging avoidance rate in the high pre-test probability cohort is consistent with the 30-35\% mediastinoscopy avoidance rate in both TBNA staging and re-staging studies $[7,8,16]$. The nonstatistical trend that SCLC was less likely to be missed by TBNA is consistent with previous studies [16].

Our overall TBNA diagnostic sensitivity of $78.7 \%$ (overall disease prevalence for malignancy $84 \%$, range 71-95\%) is comparable to the pooled sensitivity of TBNA $(78 \%)$ in a recent systematic review of TBNA studies in the field (overall disease prevalence $75 \%$, range $30-100 \%$ ) [1]. Diagnostic performance for malignancy depends on pre-test clinical probability; both accuracy and sensitivity improved substantially in patients in whom malignancy is suspected [3]. This is a well documented caveat with TBNA and has been emphasised in recent guidelines by the European Society of Thoracic Surgeons [24].

We noted our true negative TBNA results identified benign cytology but did not yield a specific benign diag-
Table 5. Cost of common lung cancer staging and diagnostic procedures

\begin{tabular}{lc}
\hline Procedure & Cost (GBP) \\
\hline Conventional TBNA & 423 \\
Mediastinoscopy & 3,008 \\
CT-guided lung biopsy & 1,311 \\
\hline
\end{tabular}

a Includes GBP 38 per disposable TBNA needle (Olympus Keymed).

nosis. This is not consistent with the literature where good results have been reported with conventional TBNA from experienced centres for the diagnosis of both sarcoidosis and tuberculosis. Trisolini et al. [21, 25] found TBNA diagnostic in $72-79 \%$ of patients with sarcoidosis in 2 studies, albeit with a histology-gauge needle and smear technique. Bilaceroglu et al. [26] obtained an $83 \%$ sensitivity for diagnosis of tuberculosis with conventional TBNA, again with a histology-gauge needle and smear technique. We attribute the failure to make a positive benign diagnosis in our study to the use of a cytology-gauge needle and the use of a liquid cytology technique causing more cytodisruption of granulomas.

Factors which may have negatively affected the overall performance of TBNA in our study include the absence of rapid on-site cytology [27], the use of a cytology rather 
Table 6. Cost analysis of diagnostic pathway for 79 patients with TBNA (TBNA+) and predicted calculation without TBNA (TBNA-)

\begin{tabular}{|c|c|c|c|c|c|c|}
\hline Criterion & TBNA+ & TBNA- & TBNA+ & TBNA- & TBNA+ & TBNA- \\
\hline Total cost saving of TBNA (GBP) & 44,220 & & 11,055 & & 33,165 & \\
\hline Mean cost (GBP/patient) & 1,068 & 1,627 & 1,349 & 1,640 & 806 & 1,615 \\
\hline Mean cost saving of TBNA (GBP/patient) & 559 & & 291 & & 809 & \\
\hline
\end{tabular}

than a histology-gauge needle [28], liquid cytology rather than smear technique [19-21] and nodal mobility [11]. The proximity of major vessels to the left paratracheal area and the more acute angulation of the TBNA needle may also have contributed to the inferior performance of paratracheal TBNA, although this is not supported by other studies [5]. The failure to surgically stage 11 of the 13 patients (Results) where a false negative TBNA result for malignancy occurred, may have also reduced the diagnostic performance. We have assumed that enlarged mediastinal nodes on CT were malignant in the presence of a primary tumour, but CT has a limited specificity for malignancy [29].

Our data also confirm experienced clinicians accurately assess pre-test clinical probability of malignancy, as the proportion of malignant cases was far higher in the high probability cohort. The lower negative predictive value of TBNA in the high probability cohort represents the higher disease prevalence. The high proportion of patients in our study in whom useful staging information was obtained is consistent with other studies demonstrating the staging utility of conventional TBNA in over $40 \%$ of the patients [30, 31].

We have demonstrated that TBNA is cost saving under the NHS's Payment by Results accountancy system by an estimated GBP 44,220 overall or GBP 559 per patient episode. This figure is likely to be conservative as a proportion of mediastinoscopy patients require overnight admission (mean length of stay 1.5 days in one UK tertiary institution [32]) which was not factored into the cost analysis. However, Payment by Results-related revenue is not proportional to innovation [33]. The flexible bronchoscopy tariff (GBP 647 according to National Tariff $2007 / 8$ [23]) is the same as that for conventional TBNA and endobronchial ultrasound-guided TBNA (EBUSTBNA) [34], both of which require more time and expertise, especially the latter. In addition, errors occur in coding the Healthcare Resource Group, especially in tertiary

Conventional Transbronchial Needle Aspiration centres, with the performance of interventional procedures leading to further inaccuracies $[33,35,36]$.

TBNA offers a day-case low-morbidity procedure, without general anaesthetics, which is more acceptable [6] and more accessible to patients than mediastinoscopy and is without the risk, albeit small, of major complications [37]. By reducing the number of absolute mediastinoscopies by 20 for 79 cases, there are additional bed saving opportunities as mediastinoscopy patients have a mean (SE) stay of 1.5 (0.18) days [32]. This would also increase thoracic surgical and theatre capacity.

We acknowledge the limitations of this study. Our study population contained a high prevalence of advanced $\mathrm{N} 2$ disease and it is acknowledged that the primary role of TBNA in patients with advanced N2 disease is for diagnosis rather than staging as radical treatment will not be appropriate. In the stage 4 patients, we accept that the role of TBNA here was for diagnosis rather than staging but this obviated the need for more invasive sampling of the metastatic site. This is a single-centre study with only subcarinal and paratracheal stations sampled. However, we did not select only for these N2 stations specifically and did not introduce selection bias here, although we acknowledge the limited number of N2 nodal locations as a limitation. We cannot comment on the exact size of the lymph nodes and success of TBNA but we acknowledge this would be relevant (all nodes were between 1 and $2 \mathrm{~cm}$ in size). We did not validate all positive TBNA results with mediastinoscopy; therefore, a false positive TBNA result is possible, although in 3 previous series where this has been evaluated the average false positive rate has been 7\% [1]. All 3 operators were technically proficient and experienced TBNA bronchoscopists, who remained unchanged for the duration of the study, thus operator-related factors are unlikely to have contributed to the differential performance at paratracheal nodes. Data confirm TBNA has a short learning curve [5]. 
We conclude that our TBNA service is safe, minimally invasive and has a high diagnostic sensitivity and accuracy for subcarinal and paratracheal nodes in selected patients with a high pre-test clinical probability of malignancy. It also provides useful staging information in $82 \%$ of the patients, confirming unsuitability for radical treatment in most but down stages $8 \%$ so that they may undergo surgical resection (although mediastinoscopy should be performed to confirm any negative TBNA result due to its superior negative predictive value [1]). TBNA obviates the need for mediastinoscopy in at least $25 \%$ (up to $37 \%$ if selecting those with a high probability of cancer) of patients, increasing thoracic surgical capacity. Our service offers a reasonable throughput (average 50 patients per year currently) which allows maintenance of technical skills and cost savings of approximately GBP
560 per patient. We therefore believe our data support continuing to perform conventional TBNA (in the absence of EBUS-TBNA) at flexible bronchoscopy when malignancy is suspected with mediastinal adenopathy, even if mediastinoscopy is available on site. In the UK, financial issues are likely to slow the development of EBUS-TBNA until the National Tariff is reviewed to reflect this innovation. Until then, we would advocate the use of conventional TBNA in all NHS respiratory units.

\section{Acknowledgment}

We would like to thank the staff in the Cytology and Endoscopy Departments at Glenfield Hospital for their contribution.

\section{References}

1 Detterbeck FC, Jantz MA, Wallace M, Vansteenkiste J, Silvestri GA; American College of Chest Physicians: Invasive mediastinal staging of lung cancer: ACCP evidencebased clinical practice guidelines, ed. 2 . Chest 2007;132(3 suppl):202S-220S.

$>2$ Cetinkaya E, Yildiz P, Altin S, Yilmaz V: Diagnostic value of transbronchial needle aspiration by Wang 22-gauge cytology needle in intrathoracic lymphadenopathy. Chest 2004; 125:527-531.

$>3$ Holty JE, Kuschner WG, Gould MK: Accuracy of transbronchial needle aspiration for mediastinal staging of non-small cell lung cancer: a meta-analysis. Thorax 2005;60: 949-955.

$\checkmark 4$ Le Jeune I, Baldwin D: Measuring the success of transbronchial needle aspiration in everyday clinical practice. Respir Med 2007; 101:670-675.

$>5$ Hermens FH, Limonard GJ, Termeer R, van den Berg W, Visser FJ, Hol BE, Janssen JP: Learning curve of conventional transbronchial needle aspiration in pulmonologists experienced in bronchoscopy. Respiration 2008;75:189-192.

6 Bernasconi M, Chhajed PN, Muller P, Borer $\mathrm{H}$ : Patients' satisfaction with flexible bronchoscopy in a hospital-based community practice. Respiration 2009;78:440-445. DOI: 10.1159/000228906.

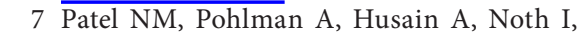
Hall JB, Kress JP: Conventional transbronchial needle aspiration decreases the rate of surgical sampling of intrathoracic lymphadenopathy. Chest 2007;131:773-778.
8 Kunst PW, Lee P, Paul MA, Senan S, Smit EF: Restaging of mediastinal nodes with transbronchial needle aspiration after induction chemoradiation for locally advanced nonsmall cell lung cancer. J Thorac Oncol 2007; 2:912-915.

-9 Bilaceroglu S, Chhajed P, Ahmet M, Aydogdu Z, Cagirici U: Computed tomographyguided transthoracic needle aspiration in diagnosing and staging hilar and mediastinal masses of lung cancer after negative bronchoscopy. J Bronchol 2007;14:149-155.

10 Yasufuku K, Chiyo M, Koh E, Moriya Y, Iyoda A, Sekine Y, Shibuya K, Iizasa T, Fujisawa T: Endobronchial ultrasound guided transbronchial needle aspiration for staging of lung cancer. Lung Cancer 2005;50:347-354.

11 Piet AH, Lagerwaard FJ, Kunst PW, van Sornsen de Koste JR, Slotman BJ, Senan S: Can mediastinal nodal mobility explain the low yield rates for transbronchial needle aspiration without real-time imaging? Chest 2007;131:1783-1787.

12 van Sornsen de Koste JR, Lagerwaard FJ, Nijssen-Visser MR, Schuchhard-Schipper R, Joosten $\mathrm{H}$, Senan S: What margins are necessary for incorporating mediastinal nodal mobility into involved-field radiotherapy for lung cancer? Int J Rad Oncol Biol Phys 2002; 53:1211-1215.

$>13$ Jenkins P, Salmon C, Mannion C: Analysis of the movement of calcified lymph nodes during breathing. Int J Rad Oncol Biol Phys 2005;61:329-334.

14 Senan S, De Ruysscher D, Giraud P, Mirimanoff R, Budach V: Literature-based recommendations for treatment planning and execution in high-dose radiotherapy for lung cancer. Radiother Oncol 2004;71:139-146.
15 Rodriguez de Castro F, Diaz Lopez F, Serda GJ, Lopez AR, Gilart JF, Cabrera Navarro P: Relevance of training in transbronchial fineneedle aspiration technique. Chest 1997;111: 103-105.

$\checkmark 16$ Hsu LH, Liu CC, Ko JS: Education and experience improve the performance of transbronchial needle aspiration: a learning curve at a cancer center. Chest 2004;125:532-540.

17 Diacon AH, Schuurmans MM, Theron J, Brundyn K, Louw M, Wright CA, Bolliger CT: Transbronchial needle aspirates: how many passes per target site? Eur Respir J 2007;29:112-116.

18 Smyth CM, Stead RJ: Survey of flexible fibreoptic bronchoscopy in the United Kingdom. Eur Respir J 2002;19:458-463.

19 Bilaceroglu S, Chhajed P: Transbronchial needle aspiration: a diagnostic tool in routine bronchoscopy. J Assoc Physicians India 2005;53:797-802.

20 Bilaceroglu S, Cagiotariotaciota U, Gunel O, Bayol U, Perim K: Comparison of rigid and flexible transbronchial needle aspiration in the staging of bronchogenic carcinoma. Respiration 1998;65:441-449.

21 Trisolini R, Agli LL, Cancellieri A, Poletti V, Tinelli C, Baruzzi G, Patelli M: The value of flexible transbronchial needle aspiration in the diagnosis of stage I sarcoidosis. Chest 2003; 124:2126-2130.

22 Department of Health 2002: Reforming NHS financial flows: introducing Payment by Results. London, DOH, 2002.

23 Department of Health: Payment by Results: Tariff Information 2007-2008. www.dh.gov. $\mathrm{uk} / \mathrm{en} / \mathrm{Managing}$ yourorganisation/Financeandplanning/NHSFinancialReforms/ DH_077279 (accessed December 2, 2009). 
24 De Leyn P, Lardinois D, Van Schil PE, RamiPorta R, Passlick B, Zielinski M, Waller DA, Lerut T, Weder W: ESTS guidelines for preoperative lymph node staging for non-small cell lung cancer. Eur J Cardiothorac Surg 2007;32:1-8.

-25 Trisolini R, Tinelli C, Cancellieri A, Paioli D, Alifano M, Boaron M, Patelli M: Transbronchial needle aspiration in sarcoidosis: yield and predictors of a positive aspirate. J Thor Cardiovasc Surg 2008;135:837-842.

26 Bilaceroglu S, Gunel O, Eris N, Cagirici U, Mehta AC: Transbronchial needle aspiration in diagnosing intrathoracic tuberculous lymphadenitis. Chest 2004;126:259-267.

-27 Diacon AH, Schuurmans MM, Theron J, Louw M, Wright CA, Brundyn K, Bolliger CT: Utility of rapid on-site evaluation of transbronchial needle aspirates. Respiration 2005;72:182-188.

28 Schenk DA, Chambers SL, Derdak S, Komadina KH, Pickard JS, Strollo PJ, Lewis RE, Patefield AJ, Henderson JH, Tomski SM, et al: Comparison of the Wang 19-gauge and 22-gauge needles in the mediastinal staging of lung cancer. Amer Rev Respir Dis 1993; 147:1251-1258.
29 Silvestri GA, Gould MK, Margolis ML, Tanoue LT, McCrory D, Toloza E, Detterbeck F; American College of Chest Physicians: Noninvasive staging of non-small cell lung cancer: ACCP evidenced-based clinical practice guidelines, ed 2. Chest 2007;132(3 suppl):178S-201S.

30 Shah PL, Singh S, Bower M, Livni N, Padley S, Nicholson AG: The role of transbronchial fine needle aspiration in an integrated care pathway for the assessment of patients with suspected lung cancer. J Thorac Oncol 2006; 1:324-327.

31 Win T, Stewart S, Groves AM, Pepke-Zaba J, Laroche CM: The role of transbronchial needle aspiration in the diagnosis of bronchogenic carcinoma. Respir Care 2003;48:602605.

32 Medford AR, Agrawal S, Free CM, Bennett JA: Mediastinal staging procedures in lung cancer: EBUS, TBNA and mediastinoscopy. Curr Opin Pulm Med 2009;15:334-342.
33 Audit Commission: PbR Data Assurance Framework 2007/08: findings from the first year of the national clinical coding audit programme. www.audit-commission.gov.uk/ nationalstudies/health/pbr/Pages/pbrdataassuranceframework200708.aspx (accessed December 2, 2009).

34 Medford AR: Endobronchial ultrasoundtransbronchial needle aspiration and lung cancer. J R Coll Physicians Edinb 2009;39: 91.

35 Medford AR, Agrawal S, Free CM, Bennett JA: Retrospective analysis of Healthcare Resource Group coding allocation for local anaesthetic video-assisted 'medical' thoracoscopy in a UK tertiary respiratory centre. QJM 2009;102:329-333.

36 Medford AR, Agrawal S, Free CM, Bennett JA: A performance and theoretical cost analysis of endobronchial ultrasound-guided transbronchial needle aspiration in a UK tertiary respiratory centre. QJM 2009;102:859864. DOI: $10.1093 /$ qjmed/hcp136.

- 37 Puhakka HJ: Complications of mediastinoscopy. J Laryng Otol 1989;103:312-315. 